\title{
Nonequilibrium phenomena and determination of plasma parameters in the hot core of the cathode region in free-burning arc discharges
}

\author{
Gerrit Kühn* ${ }^{\dagger} \dagger$ \\ Institut für Atom- \& Molekülphysik, Abteilung Plasmaphysik, Universität Hannover, Germany Institut für Gravitationsphysik, Universität \\ Hannover, Germany \\ and Max-Planck-Institut für Gravitationsphysik, Albert-Einstein-Institut, Hannover, Germany \\ Manfred Kock \\ Institut für Atom- \& Molekülphysik, Abteilung Plasmaphysik, Universität Hannover, Germany
}

(Received 4 July 2006; published 19 January 2007)

\begin{abstract}
We present spectroscopic measurements of plasma parameters (electron density $n_{\mathrm{e}}$, electron temperature $T_{\mathrm{e}}$, gas temperature $T_{\mathrm{g}}$, underpopulation factor $b$ ) in the hot-core region in front of the cathode of a low-current, free-burning arc discharge in argon under atmospheric pressure. The discharge is operated in the hot-core mode, creating a hot cathode region with plasma parameters similar to high-current arcs in spite of the fact that we use comparatively low currents (less than $20 \mathrm{~A}$ ). We use continuum emission and (optically thin) line emission to determine $n_{\mathrm{e}}$ and $T_{\mathrm{e}}$. We apply relaxation measurements based on a power-interruption technique to investigate deviations from local thermodynamic equilibrium (LTE). These measurements let us determine the gas temperature $T_{\mathrm{g}}$. All measurements are performed side-on with charge-coupled-device cameras as detectors, so that all measured plasma parameters are spatially resolved after an Abel inversion. This yields the first ever spatially resolved observation of the non-LTE phenomena of the hot core in the near-cathode region of free-burning arcs. The results only partly coincide with previously published predictions and measurements in the literature.
\end{abstract}

DOI: 10.1103/PhysRevE.75.016406

PACS number(s): 52.80.Mg, 52.70.-m, 32.70.Fw, 52.20.Fs

\section{INTRODUCTION}

The hot-core region in front of the cathode of freeburning arcs has been under investigation for several decades, in both experimental and theoretical works (see, e.g., [1-14]). Knowledge about the boundary layers close to the cathode is crucial for an understanding of the arc-to-cathode attachment and thus of the whole discharge.

Our discharge under investigation is a low-current (1-20 A) free-burning arc operated in argon under atmospheric pressure. The discharge was developed to serve as a prototype for high-intensity discharge (HID) lamps, which are used, e.g., in video beamers, cinema projectors, or automotive headlights. Compared to these commercially available lamps, our model lamp benefits from a flexible design, which allowed us to vary parameters like the material and shape of the electrodes, the arc length, the filling gas, and the gas pressure. Furthermore, the discharge vessel features view ports with windows of high optical quality allowing quantitative spectroscopy with high accuracy. It is thus especially suited for fundamental research.

In previous experiments we found a special discharge mode we called the "blue-core" mode. This mode features a very hot plasma zone in front of the cathode $[15,16]$. Therefore we changed the name to the more general term "hot core." Figure 1 shows pictures of the cathode region for the

\footnotetext{
*Electronic address: gerrit@pmp.uni-hannover.de; URL: http:// www.plasmaphysik.eu/

${ }^{\dagger}$ Address to be used for correspondence: Albert-Einstein-Institut, Callinstraße 38, D-30167 Hannover, Germany.
}

hot-core mode in comparison with the well-known diffuse mode. The plasma parameters (such as $n_{\mathrm{e}}$ and $T_{\mathrm{e}}$ ) are similar to those obtained on high-current arc discharges or plasma jets. Our group as well as other researchers have reported deviations from local thermodynamic equilibrium (LTE) in these types of plasmas. There is an ongoing discussion about the origin of these deviations and whether the experimental techniques of determination are accurate. Especially the applicability of Thomson scattering for these plasmas has been questioned lately [17-22].

Measurements in the near-cathode region are challanging: One needs a detector with both high spatial resolution and high dynamic range to be able to look into the small boundary layers and to overcome steep gradients in temperature and particle density. Furthermore, the validity of many methods of classical plasma spectroscopy becomes arguable if the plasma is not in LTE.

We used a partial LTE (PLTE) model to describe the deviations from the LTE state (see Sec. II). Our experimental methods and data evaluation procedures (described in Secs. III and IV) take these deviations into account. Apart from passive emission spectroscopy we employ a spatially resolved relaxation experiment to determine PLTE parameters in this kind of discharge. The results presented in Sec. V thus give a spatially resolved, quantitative insight into the plasma parameters and hence their deviations from the LTE state in the near-cathode region of the hot core in free-burning arcs.

\section{THEORETICAL BACKGROUND: THE PLTE PLASMA MODEL}

We adopted our PLTE plasma model used in this publication from pioneer work done by Richter $[23,24]$. In the 

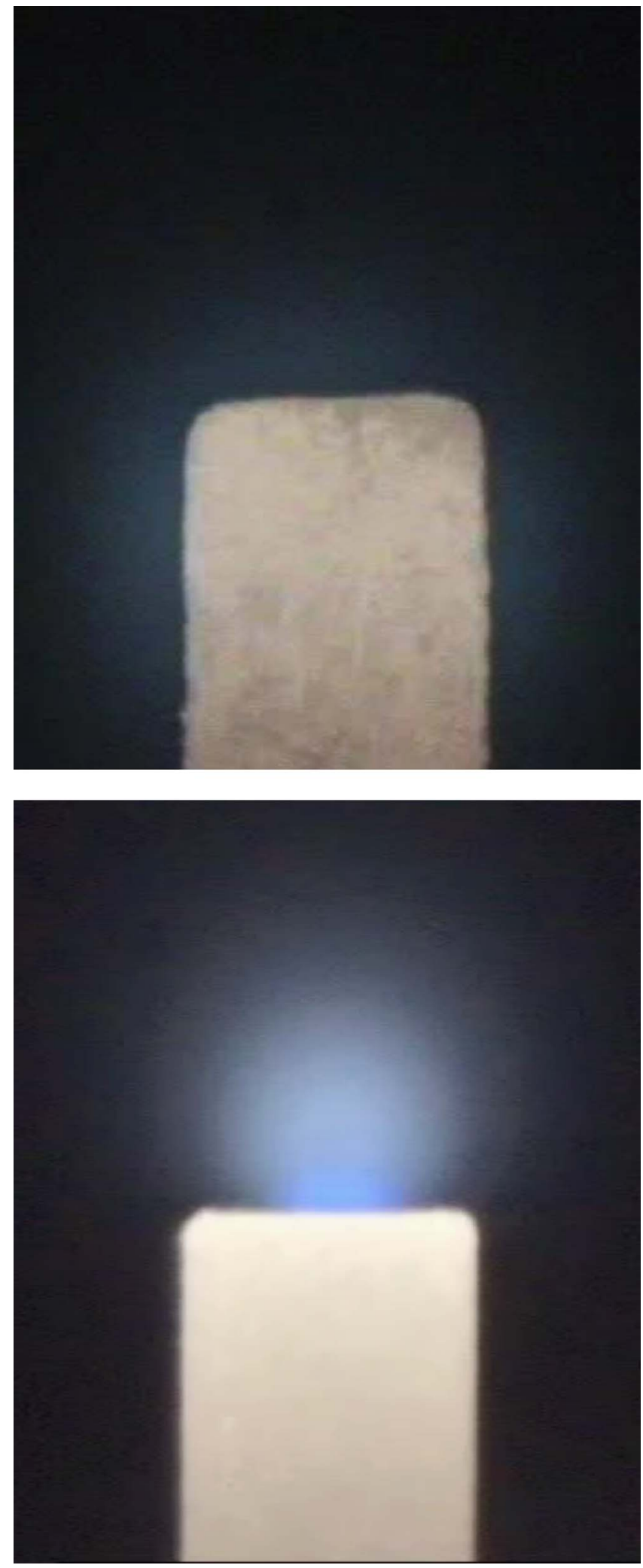

FIG. 1. (Color online) Pictures of the cathode region in the diffuse mode (left) and the hot-core mode (right). The cathode diameter was $0.6 \mathrm{~mm}$ and the arc current was $12.5 \mathrm{~A}$ in both cases.

following we give an overview of the basic assumptions and consequences of the model. A more detailed description can be found in [25].
The LTE state can be described with four basic equations: Dalton's law, the quasineutrality condition, the Saha equation, and the Boltzmann distribution. For a plasma of known chemical composition these equations leave one free parameter in the model-e.g., $T_{\mathrm{e}}$ or $n_{\mathrm{e}}$. Either parameter can be chosen for calculations to parametrize the model. However, one must be aware of ambiguities; e.g., there can be more than one possible electron density $n_{\mathrm{e}}$ for a given temperature $T_{\mathrm{e}}$.

To reach the LTE condition, collisions have to dominate the plasma. An electric arc burning in argon gains energy from the applied electric field. This energy is transferred almost exclusively to the free electrons in the discharge, due to their high mobility. Collisional processes spread the energy among the electrons and between the electrons and the heavy particles. A common kinetic temperature $T_{\mathrm{e}}$ for the electrons is established comparatively easily because of high collision rates between free electrons with high-momentum transfer cross sections. If the (elastic) collision rates are too low to establish a common temperature, the first deviation from LTE is a drop of the kinetic gas temperature $T_{\mathrm{g}}$ of the heavy particles below $T_{\mathrm{e}}$. Furthermore, all atoms of noble gases feature a separation of the ground state from all excited states by a relatively large energy gap. All excited levels of, e.g., Ar I have energies above $70 \%$ of the ionization energy. Low collision rates lead to a deviation from the Boltzmann distribution, which first of all becomes manifest in an underpopulation of the excited levels compared to the ground state, because the inelastic cross section for collisions with electrons is markedly lower for the ground state than for the excited states.

These considerations lead to the introduction of two additional parameters to describe a plasma with some deviations from LTE, which we call the PLTE state: (i) the gas temperature $T_{\mathrm{g}} \leqslant T_{\mathrm{e}}$ and (ii) the underpopulation factor $b \leqslant 1$.

$T_{\mathrm{g}}$ modifies Dalton's law to read

$$
p=k_{\mathrm{B}}\left(n_{\mathrm{e}} T_{\mathrm{e}}+\sum_{i=0}^{N_{\mathrm{Ar}}} n_{i} T_{\mathrm{g}}\right),
$$

where $p$ denotes the pressure of the discharge, $N_{\mathrm{Ar}}$ the maximum ionization stage of argon to take into account, and $n_{i}$ the density for ionization stage $i$. Sometimes it is more convenient to use the ratio $\beta:=T_{\mathrm{e}} / T_{\mathrm{g}} \geqslant 1$ instead of $T_{\mathrm{g}}$. The underpopulation factor $b$ enters the Saha-Boltzmann relation to lower all excited state densities. This can be achieved by replacing the statistical weights $g_{0}^{|\nu\rangle}$ for all excited $(\nu>0)$ levels of Ar I with $b g_{0}^{|\nu\rangle}$; all ion densities are then underpopulated, because they are in partial Saha equilibrium with the excited states of Ar I.

The PLTE plasma model is successfully applied in literature to describe deviations from LTE in arc discharges (see, e.g., [25-29]). Because there are three free parameters (instead of only one in the LTE case), a plasma in PLTE is more difficult to determine experimentally. The LTE model features one free parameter (given a known plasma composition), which can in principle be determined with a single spectroscopic measurement like an absolute intensity 


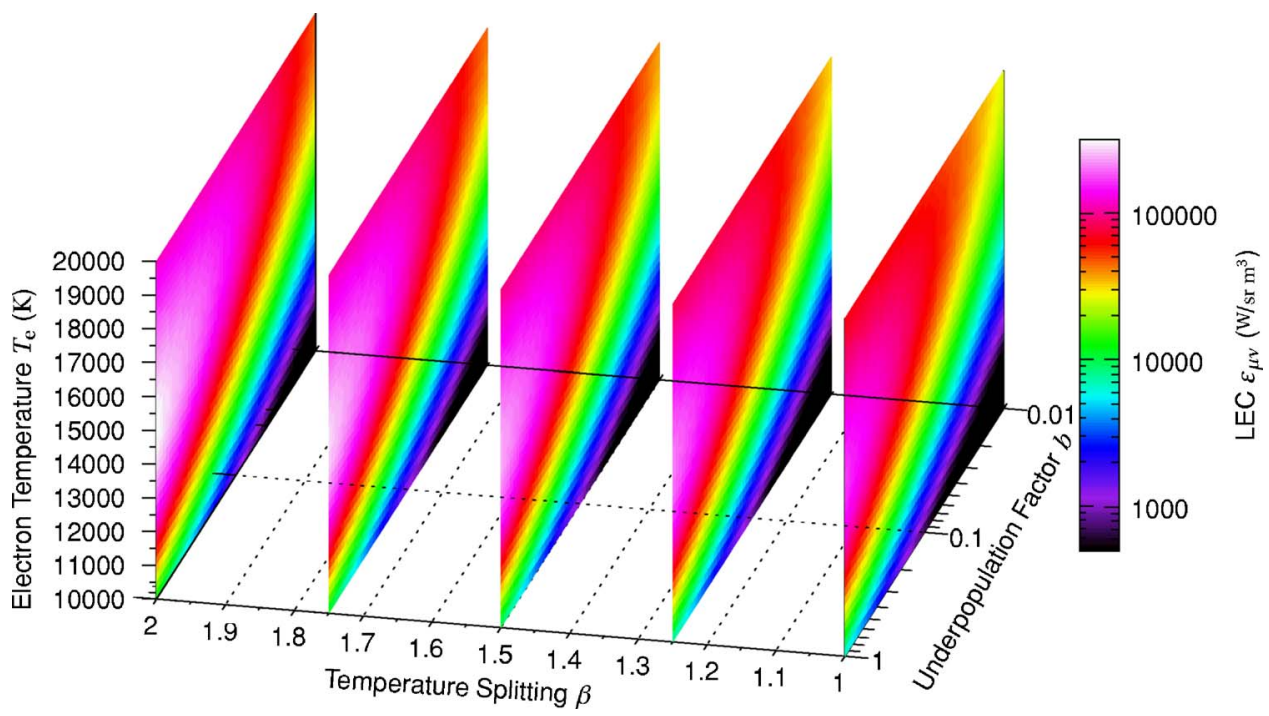

FIG. 2. (Color online) Line emission coefficient (LEC) of the Ar I line at $7147.04 \AA$ in a PLTE plasma in dependence of the electron temperature $T_{\mathrm{e}}$, the temperature splitting $\beta$, and the underpopulation factor $b$. of an optically thin line. The PLTE model as described above has three free parameters; thus, we need to measure three independent quantities to determine the plasma state.

To obtain an overview of the PLTE plasma model and the impact of the additional parameters, Fig. 2 shows calculated line emission coefficients (LEC's) with $T_{\mathrm{e}}, \beta$, and $b$ as parameters in a three-dimensional pseudocolor plot. Additional plots showing the dependence of the LEC on two parameters (keeping the third constant) can be found in $[25,30]$. By design of the model, for $\beta=1$ and $b=1$ the LTE values for the LEC are reproduced. Furthermore, we note that for given values of $T_{\mathrm{e}}$ and $\beta$ a decreasing value of $b$ (corresponding to an increasing underpopulation) leads to a decreasing line emission, whereas for given values of $T_{\mathrm{e}}$ and $b$ an increasing value of $\beta$ (corresponding to an increasing difference between $T_{\mathrm{e}}$ and $T_{\mathrm{g}}$ ) leads to an increasing line emission. Figure 2 also shows that the PLTE model can only be described unambiguously using three independent parameters. For each LEC (color or grayscale level) there is a hyperplane containing an arbitrary number of parameter combinations $\left(T_{\mathrm{e}}, \beta, b\right)$ leading to this particular LEC. Even a known temperature $T_{\mathrm{e}}$ and a measured LEC leaves us with a curve of possible plasma states. Consequently, we have to determine three independent parameters experimentally to be able to specify an unambiguous plasma state.

For calculation purposes it is useful to parametrize the model using the parameter set $\left(T_{\mathrm{e}}, \beta, b\right)$. However, we used the mathematically equivalent set $\left(T_{\mathrm{e}}, n_{\mathrm{e}}, \beta\right)$ for the determination of the plasma state, because $n_{\mathrm{e}}$ is much easier to determine experimentally than $b$ (see Sec. IV).

\section{EXPERIMENTAL SETUP}

We used a combination of passive and active emission spectroscopy for the determination of the plasma parameters. Figure 3 shows our experimental setup, which has been described in detail elsewhere [25]. We observed the arc side-on using the established $\mathrm{T}$ arrangement for quantitative spectroscopy. The turnable mirror, which is located at the intersection of the "T," is also the lower part of a mirror tower

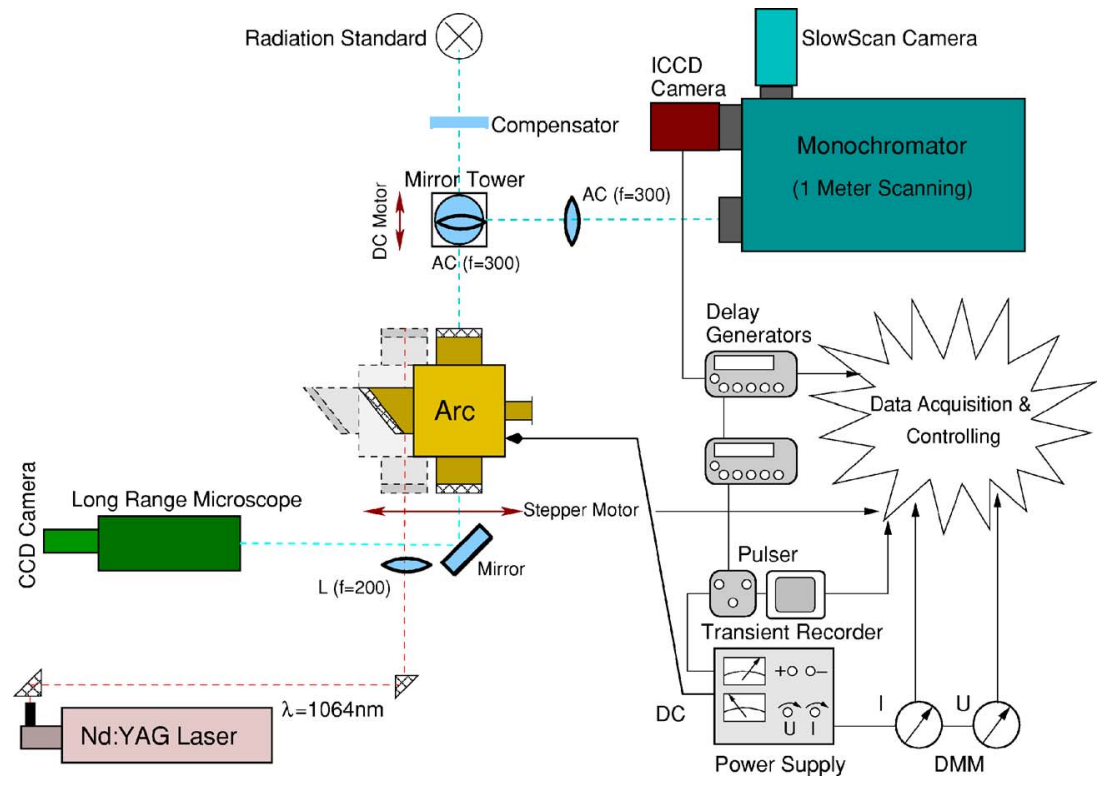

FIG. 3. (Color online) Overview of the experimental setup for active and passive emission spectroscopy on the free-burning arc. 


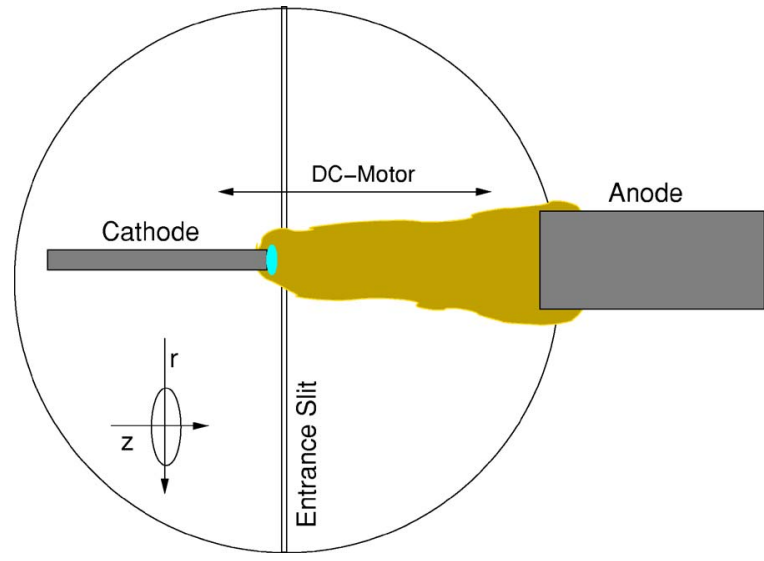

FIG. 4. (Color online) Mapping of the rotated image of the arc plasma onto the entrance slit of the monochromator.

that we used to rotate the image of the arc by $90^{\circ}$ before mapping it onto the entrance slit of a 1-m monochromator (see Fig. 4). Furthermore, we were able to move this mirror with the aid of a PC-controlled dc motor to select the plasma region in front of the cathode to be measured.

The slit width of the monochromator (and thus also the spatial resolution of the experiment) was set to $50 \mu \mathrm{m}$. The distance of the electrodes was set to $10 \mathrm{~mm}$, and their length above the holders was $10 \mathrm{~mm}$ as well. The cathode was a rod of thoriated $(1.8 \% \mathrm{Th})$ tungsten $(0.6 \mathrm{~mm}$ in diameter), and the anode was a rod of pure tungsten ( $2 \mathrm{~mm}$ in diameter). In the exit plane of the monochromator we used a slow-scan charge-coupled-device (CCD) camera for classical emission spectroscopy and an iCCD camera to detect transient plasma phenomena during the relaxation measurements. The actual detector could be chosen via a turnable mirror. See Table I for further technical data of the two detector systems.

The discharge was operated in argon (purity better than 99.9993\%) under atmospheric pressure in an upright position with the anode on top and the cathode below. We used a pulsed Nd:YAG laser to ignite the discharge at comparatively low voltages (less than $250 \mathrm{~V}$ ) by means of a laserinduced optical breakdown. This method of ignition provided various advantages concerning the construction and handling of the discharge [25].

We carried out the relaxation experiments by introducing a short-circuit in parallel with the discharge for about $20 \mu \mathrm{s}$ with the help of a Power metal-oxide-semiconductor

TABLE I. Technical details of the CCD camera systems used for spectroscopy.

\begin{tabular}{lcc}
\hline \hline & Slow-scan CCD & Intensified CCD \\
\hline Model & PI LN/CCD-1752-PF & RS PI-MAX-1024-E \\
Cooling & $-125^{\circ} \mathrm{C}\left(\mathrm{lN}_{2}\right)$ & $-40^{\circ} \mathrm{C}($ Peltier $)$ \\
Pixel size & $15 \mu \mathrm{m} \times 15 \mu \mathrm{m}$ & $28 \mu \mathrm{m} \times 28 \mu \mathrm{m}$ \\
Chip size & $1752 \mathrm{px} \times 532 \mathrm{px}$ & $1024 \mathrm{px} \times 256 \mathrm{px}$ \\
A/D range & $18 \mathrm{Bit}$ & $16 \mathrm{Bit}$ \\
Spectral range & $2200 \AA-11000 \AA$ & $5000 \AA-9000 \AA$ \\
Exposure time & $\geqslant 80 \mathrm{~ms}($ shutter $)$ & $\geqslant 5 \mathrm{~ns}(\mathrm{MCP})$ \\
\hline \hline
\end{tabular}

TABLE II. Argon line data used in this work.

\begin{tabular}{lcccccc}
\hline \hline Species & $\lambda_{\mu \nu}(\AA)$ & $E^{|\mu\rangle}\left(\mathrm{cm}^{-1}\right)$ & $g_{\mu}$ & $A_{\mu \nu}\left(10^{6} \mathrm{~Hz}\right)$ & Err. (\%) & Ref. \\
\hline Ar I & 4510.73 & 117563 & 1 & 1.18 & 7 & {$[31]$} \\
Ar I & 4522.32 & 116660 & 3 & 0.0898 & 8 & {$[31]$} \\
Ar I & 7147.04 & 107132 & 3 & 0.625 & 8 & {$[31]$} \\
Ar II & 4400.1 & 155351 & 4 & 16.0 & 11 & {$[32]$} \\
Ar II & 4401.0 & 155043 & 6 & 30.4 & 8 & {$[32]$} \\
Ar II & 4420.9 & 155351 & 4 & 3.1 & 11 & {$[32]$} \\
Ar II & 4426.0 & 157673 & 6 & 81.7 & 5 & {$[32]$} \\
Ar II & 4430.2 & 158168 & 4 & 56.9 & 4 & {$[32]$} \\
Ar II & 4431.0 & 155043 & 6 & 10.9 & 11 & {$[32]$} \\
Ar II & 4433.84 & 194883 & 8 & 64.98 & 10 & {$[33]$} \\
\hline \hline
\end{tabular}

field-effect transistor (MOSFET) connected in parallel to the power supply. After these $20 \mu$ s the current was switched on again, so the discharge could continue operation. Diodes, MOSFET, resistors, and additional electronics were chosen and designed for switching times less than $250 \mathrm{~ns}$. For a detailed description of the electronics for the relaxation experiment see $[25,30]$.

\section{DATA ACQUISITION AND EVALUATION}

\section{A. Passive emission spectroscopy}

We used the slow-scan CCD camera to measure the emission of both $\mathrm{Ar}$ lines and the continuum on an absolute scale. A tungsten-strip lamp calibrated by PTB (German national metrology institute) served as a radiation standard. The standard detection range fitting onto the CCD chip was between $4350 \AA$ and $4550 \AA$. This part of the blue spectrum contains various $\mathrm{Ar}$ I and $\mathrm{Ar}$ II emission lines suitable for quantitative spectroscopy of lines as well as a region (4455-4456 $)$ appropriate for measuring the continuum emission.

Throughout this paper we used Ar II line emission and continuum emission for the determination of $n_{\mathrm{e}}$ and $T_{\mathrm{e}}$. This overcomes the problem of the unknown underpopulation factor in the Boltzmann distribution for Ar I and the unknown gas temperature in Eq. (1). Table II contains the atomic line data used. We checked both experimentally and mathematically that all lines were optically thin under our experimental conditions.

As stated in [25] we need two measured quantities to determine $n_{\mathrm{e}}$ and $T_{\mathrm{e}}$ from the remaining equations. Apart from Ar II line emission we used the continuum emission coefficient (CEC) of the above-mentioned spectral range, which is related to $n_{\mathrm{e}}$ and $T_{\mathrm{e}}$ [34]:

$$
\varepsilon_{\lambda}=\frac{e^{6}}{12 c^{2} \varepsilon_{0}^{3} \sqrt{6 \pi^{5} m_{\mathrm{e}}^{3} k_{\mathrm{B}}}} \frac{n_{\mathrm{e}}}{\lambda^{2} \sqrt{T_{\mathrm{e}}}} \sum_{i=1}^{N_{\mathrm{Ar}}} n_{i} i^{2} \xi_{i}\left(\lambda, T_{\mathrm{e}}\right),
$$




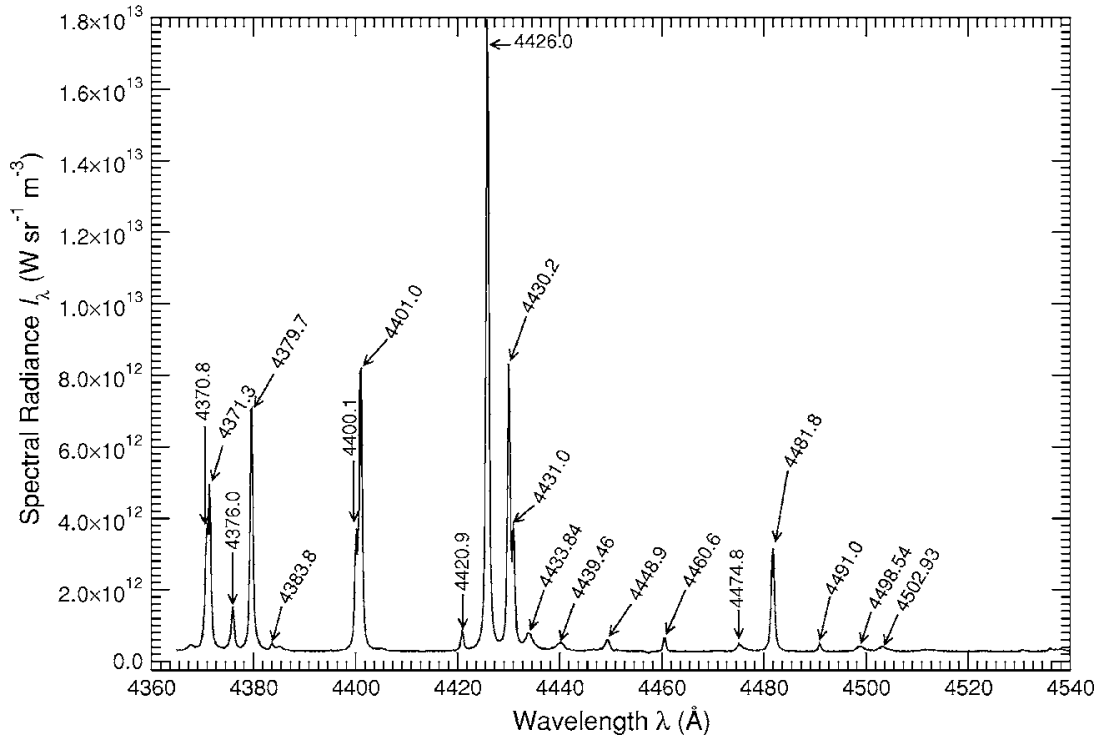

FIG. 5. Spectrum from the center of the discharge (still Abel integrated) for the first axial position $(z=50 \mu \mathrm{m})$ in front of the cathode at $I=12.5 \mathrm{~A}$. All identified lines belong to transitions of Ar II. Additional line data can be found in $[32,33]$.

$$
\begin{aligned}
\xi_{i}:= & {\left[1-\exp \left(-\frac{h c}{\lambda k_{\mathrm{B}} T_{\mathrm{e}}}\right)\right] \frac{\gamma_{i}}{Z_{i}\left(T_{\mathrm{e}}, n_{\mathrm{e}}\right)} \xi_{z}^{\mathrm{fb}}\left(\lambda, T_{\mathrm{e}}\right) } \\
& +\exp \left(-\frac{h c}{\lambda k_{\mathrm{B}} T_{\mathrm{e}}}\right) \xi_{i}^{\mathrm{ff}}\left(\lambda, T_{\mathrm{e}}\right) .
\end{aligned}
$$

Here $Z_{i}\left(T_{\mathrm{e}}, n_{\mathrm{e}}\right)$ denotes the partition function for atoms of the ionization stage $i$ and $\gamma_{i}$ represents the statistical weight of the parent ion. The other symbols have their usual meaning. The Biberman factors $\xi_{i}^{\mathrm{fb}}$ for free-bound and $\xi_{i}^{\mathrm{ff}}$ for free-free transitions of the electrons are expressed as one effective $\xi_{i}$ factor for each ionization stage $i$. We included $\xi$ factors for Ar II and Ar III (thus setting $N_{\mathrm{Ar}}=2$ ) and assumed $\xi_{1}=1.86$ (see [35-37]). For $\xi_{2}$ we extrapolated the data given by [38] to minimize systematic errors as shown in [39]. Continuum emission is especially suited for determining the electron density in PLTE plasmas, because its applicability only requires a Maxwellian distribution for the free electrons. Thus we were able to extract the data to determine $n_{\mathrm{e}}$ and $T_{\mathrm{e}}$ for the PLTE plasma state from a single CCD frame.

Figure 5 shows a typical spectrum from the center of the discharge. A peculiar feature of the hot-core mode (which previously also led to the name "blue core") is the intense Ar II line emission in the blue spectral region. Although in the diffuse mode the Ar I lines at $4511 \AA$ and $4522 \AA$ dominate the blue region shown in Fig. 5, the spectrum of the hot-core mode is completely dominated by ionic line emission, so that the Ar I lines cannot be seen against the Ar II lines and the continuum emission in Fig. 5.

To yield spatially resolved data we had to perform an Abel inversion before determining the plasma parameters. Therefore we used the inversion algorithm proposed in [40], which is based on an approximation of the unknown radial function with a Fourier series. The unknown Fourier coefficients are then determined with a least-squares fit to the measured data points. In contrast to other methods of Abel inversion we neither have to use the derivative of the measured data (which would lead to an amplification of noise) nor do we fit the measured data before the inversion process (which may tamper with the actual data). This Fourier method of Abel inversion provides very accurate inverted data [41]. Taking into account that both the Ar II lines and the continuum emission under investigation in this work showed monotonically decreasing profiles only, which are far less prone to error for Abel inversion [42], we ensured that the inverted data had high quality. The line data were always integrated spectrally before the inversion process to further improve the S/N ratio. Additionally, we used a Questar QM1 long-range microscope, equipped with a video CCD camera, to monitor and record the cathode region of the arc while the measurements were taken. Thus we made sure that the cylindrical symmetry, neccessary for the application of the Abel inversion, was always maintained. The pictures shown in Fig. 1 are fixed images from these digital video recordings.

\section{B. Active emission spectroscopy}

The relaxation method [25,43-45] is based on a fast power interruption and a measurement of the subsequent transient plasma phenomena. Deviations from LTE appear in form of an unusual development of the line emission during the power interruption. For argon plasmas not in LTE, an increase in line emission during the interruption can be measured, because excited Ar I levels stay in partial Saha equilibrium with the free electrons that cool rapidly from $T_{\mathrm{e}}$ to $T_{\mathrm{g}}$. This is the so-called Saha response $[25,43,46]$. The ratio $\Theta$ of the maximum line emission from level $|\nu\rangle\left(I_{0}^{\prime}|\nu\rangle\right)$ reached during the power interruption and the continuous line emission $\left.\left(I_{0}^{\nu}\right\rangle\right)$ is [25]

$$
\Theta:=\frac{I_{0}^{\prime|\nu\rangle}}{I_{0}^{|\nu\rangle}}=\sqrt{\beta^{3}} \exp \left(\frac{\left(E_{0}^{\infty}-\Delta E_{0}^{\infty}-E_{0}^{|\nu\rangle}\right)}{k_{\mathrm{B}} T_{\mathrm{e}}}(\beta-1)\right) .
$$

Here $E_{0}^{\infty}$ denotes the ionization energy for neutral argon, $\Delta E_{0}^{\infty}$ accounts for the lowering of the ionization energy according to [47], and $E_{0}^{|\nu\rangle}$ is the energy of the neutral argon level $|\nu\rangle$ under investigation. Thus, given values for $T_{\mathrm{e}}$ obtained from passive emission spectroscopy as described above, the relaxation method yields $\beta$ and hence $T_{\mathrm{g}}$. 


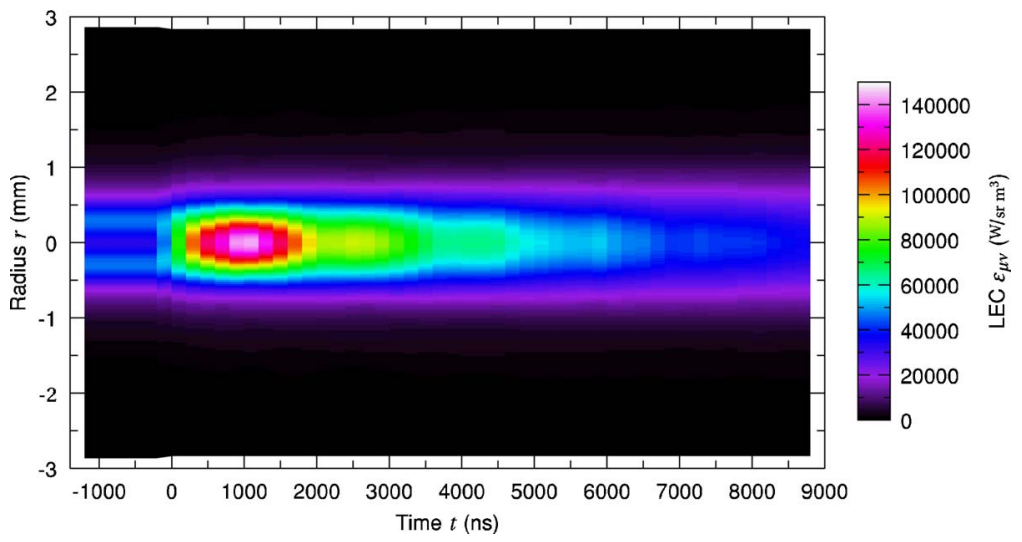

FIG. 6. (Color online) Radially and temporally resolved relaxation process following a fast power interruption. The abscissa shows the temporal evolution ( $t=0$ denotes the beginning of the power interruption) and the ordinate shows the radial resolution of the $\operatorname{Ar} I$ LEC (displayed in pseudo colors). The measurement was carried out for the first axial position $(z=50 \mu \mathrm{m})$ in front of the cathode at $I=12.5 \mathrm{~A}$. The temporal resolution (gate width) for the exposure of the iCCD camera was $200 \mathrm{~ns}$.

We recorded the relaxation process using the iCCD camera system with a typical gate width of $200 \mathrm{~ns}$, which determined our temporal resolution. The offset between the exposure of the camera and the power-interruption process could be varied with the help of two PC-controlled delay generators (DG-535 by Stanford Research). We repeated the experiment with $200 \mathrm{~Hz}$ to improve the S/N ratio. The iCCD camera system was set to sum up the repetitions on chip before reading out the averaged frame to speed up data acquisition. Typical measurements take between $1 \mathrm{~s}$ and $10 \mathrm{~s}$ for one time frame. The standard line for this diagnostic technique was the Ar I line at $7147 \AA$ (see Table II).

The evaluation of the data was similar to the previously described procedure for passive emission spectroscopy. After determining the time frame for the emission maximum, the line was integrated spectrally and subsequently Abel inverted. For the continuous line emission several time frames were averaged to further improve the $\mathrm{S} / \mathrm{N}$ ratio. For high temperatures above the so-called norm temperature [48] of about $15000 \mathrm{~K}$ this was especially important, because radial profiles with off-axis maxima are more prone to error for an Abel inversion [42]. Figure 6 shows the spatially and temporally resolved relaxation process following a power interruption.

\section{Determination of plasma parameters}

The procedure to yield the plasma parameters $\left(n_{\mathrm{e}}, T_{\mathrm{e}}, \beta\right)$ from the measured data is described in detail in [25]. After
Abel inversion we took the measured absolute LEC and CEC to simultaneously satisfy Saha's equation for the densities $n_{1}$ and $n_{2}$ of $\mathrm{Ar}$ II and $\mathrm{Ar}$ III, the quasineutrality condition, and the Boltzmann distribution. This is possible even in the PLTE case without knowing $\beta$ or $b$ and provided us with values for $n_{\mathrm{e}}$ and $T_{\mathrm{e}}$ corresponding to the measured LEC and CEC. We always evaluated jointly the measured CEC and LEC from one acquired spectrum to obtain consistent values for $n_{\mathrm{e}}$ and $T_{\mathrm{e}}$ in one run.

With $T_{\mathrm{e}}$ we were able to evaluate the relaxation experiments and to calculate $\beta$ and hence $T_{\mathrm{g}}$ according to Eq. (4). Thus we could determine a full set of plasma parameters $\left(n_{\mathrm{e}}, T_{\mathrm{e}}, \beta\right)$ for each radial position of each measured region in front of the cathode.

Electron temperatures are mainly determined by the LEC's measured for ionic line emission and can reach an accuracy better than $2 \%$. Provided that the assumptions about the plasma model are valid, we estimate the error for $T_{\mathrm{e}}$ to be always smaller than $5 \%$ and the accuracy for $n_{\mathrm{e}}$ between $5 \%$ and $10 \%$. The determination of $\beta$ and $T_{\mathrm{g}}$, respectively, is dominated by the exponential factor in Eq. (4). In principal, the uncertainty for $\Theta$ is rather low because it is the ratio of two measured quantities and the error propagation is further weakened by the exponential function. Thus, the main source of uncertainty for $\beta$ is $T_{\mathrm{e}}$. We estimate that the overall error for $\beta$ and $T_{\mathrm{g}}$ usually does not exceed $15 \%$. Thereby we already consider a larger uncertainty for the values in the center of the discharge because of the difficult

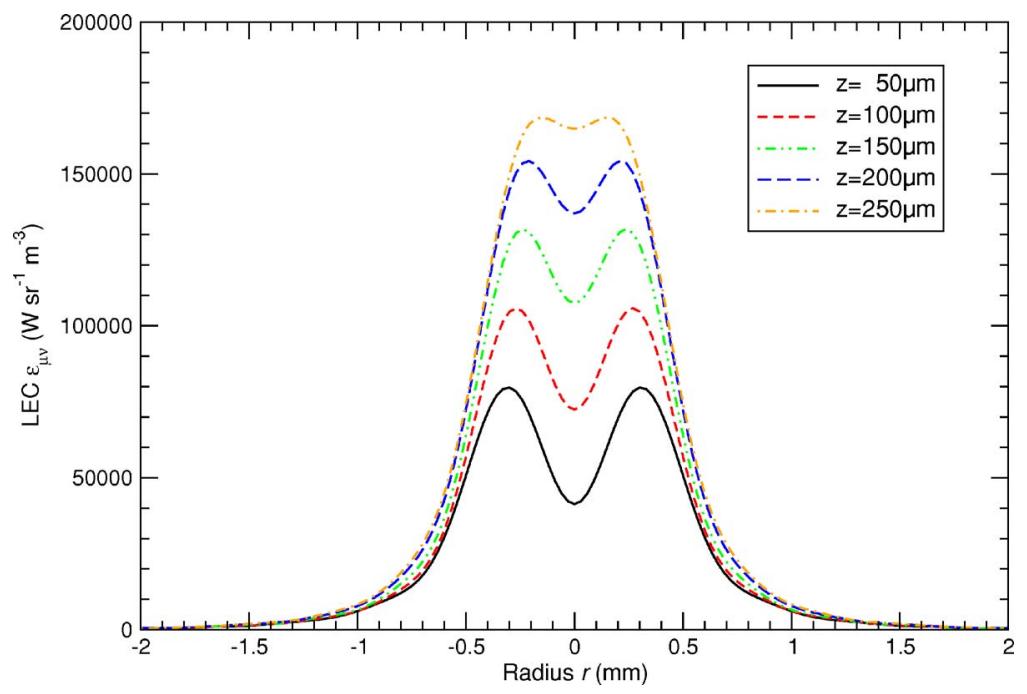

FIG. 7. (Color online) LEC for the Ar I line at $7147 \AA$ for different axial distances from the cathode after Abel inversion (measured with the slow-scan CCD camera). The arc was operated in the hot-core mode under atmospheric pressure at $I=12.5 \mathrm{~A}$. Close to the cathode the off-axis Larenz maxima appear, indicating that the center of the discharge has exceeded the norm temperature. 


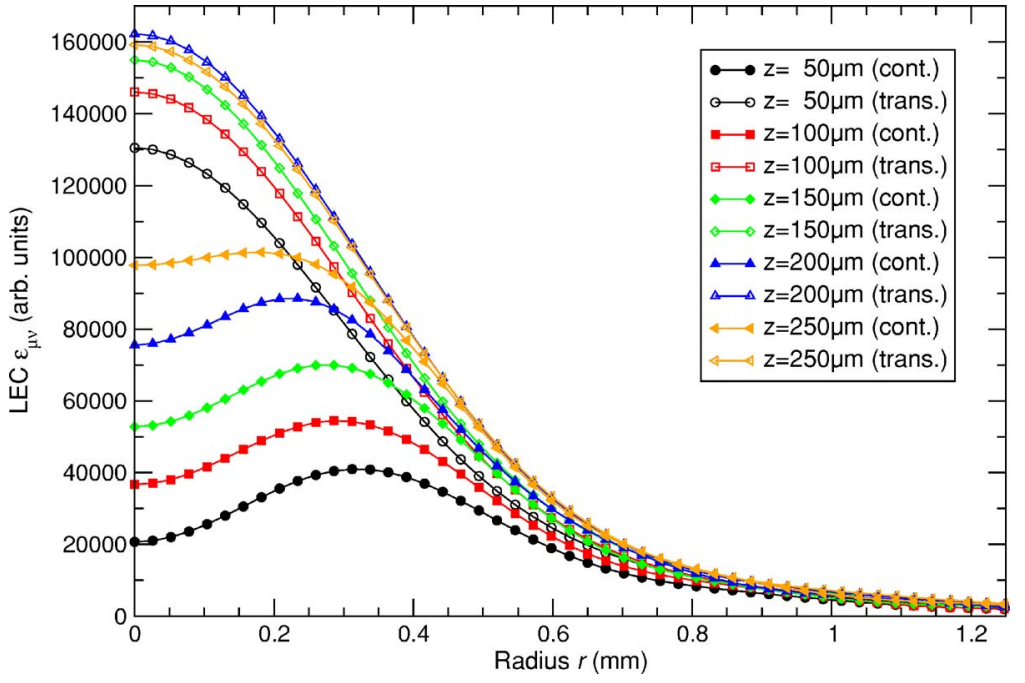

FIG. 8. (Color online) LEC's for the Ar I line at $7147 \AA$ for the first five axial positions in front of the cathode after Abel inversion (measured with the iCCD camera). The arc was operated in the hot-core mode under atmospheric pressure at $I=12.5$ A. Curves with solid symbols display emission for a continuously operated discharge, whereas open symbols display the transient emission maximum during power interruption.
Abel inversion process for profiles with off-axis maxima.

The complete set of plasma parameters allowed us to describe the state of the arc plasma in the scope of the PLTE model unambiguously. We were also able to calculate further quantities from the PLTE model. One value to determine was the underpopulation factor $b$. Furthermore, we could give an estimate for the electric field strength $E$ using a simple formula from [49]

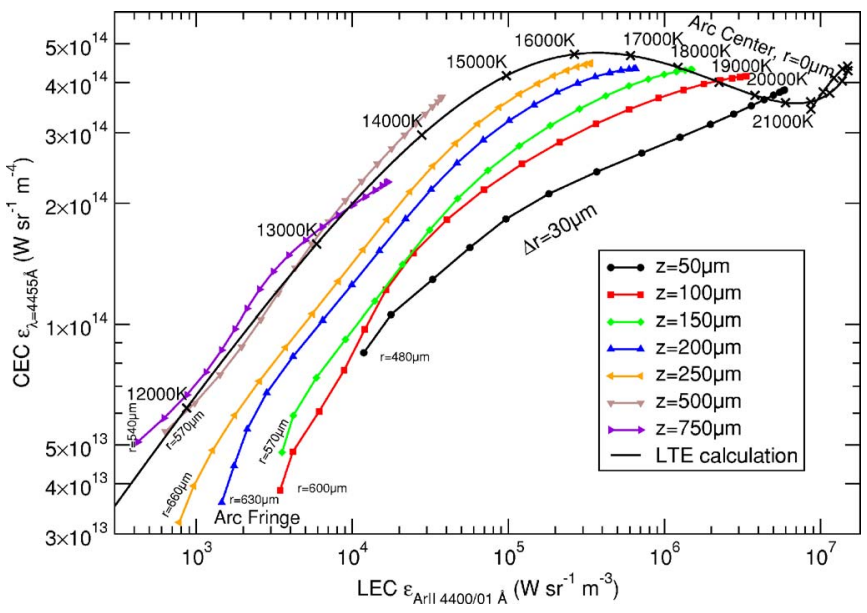

FIG. 9. (Color online) Olsen-Richter plot consisting of measured LEC's for Ar II and CEC's after Abel inversion. Here and in the following figures the notation $4400 / 01 \AA$ refers to more than one line being evaluated simultaneously (in the case the lines at $4400 \AA$ and $4401 \AA$ ). See Table II for a list of lines under investgation. The radial position $r$ and the axial position $z$ serve as implicit parameters for these measurements. The arc was operated in the hot-core mode under atmospheric pressure at $I=12.5 \mathrm{~A}$. For each axial distance a curve starts at high values stemming from the center of the discharge and then decreases radially. The distance between two measured points of a curve is $30 \mu \mathrm{m}$ due to the applied $2 \times 2$ hardware binning of the slow-scan camera. Results of an LTE calculation parametrized with temperature are displayed for comparison with the measurements.

$$
\frac{\beta-1}{\beta}=\frac{m_{\mathrm{Ar}}}{9 m_{\mathrm{e}}}\left(\frac{\lambda_{\mathrm{e}} e \vec{E}}{k_{\mathrm{B}} T_{\mathrm{e}}}\right)^{2},
$$

where $\lambda_{\mathrm{e}}$ is the mean free path length for electrons and the other symbols have their usual meaning. For $\lambda_{\mathrm{e}}$ we considered collisions with electrons, Ar I, Ar II, and Ar III, and set (according to [50])

$$
\lambda_{\mathrm{e}}:=\left(n_{\mathrm{e}}\left\langle Q_{\mathrm{ee}}\right\rangle+\sum_{i=0}^{2} n_{i}\left\langle Q_{\mathrm{e}} i\right\rangle\right)^{-1} .
$$

We obtained values for neutral argon $\left(\left\langle Q_{\mathrm{e} 0}\right\rangle\right)$ by cubic spline interpolation of the data published in [51], whereas we calculated the values for ions $\left(\left\langle Q_{\mathrm{e} 1}\right\rangle\right.$ for $\mathrm{Ar}$ II and $\left\langle Q_{\mathrm{e} 2}\right\rangle$ for Ar III) according to [50] (Chap. II, Sec. 8). Finally, we assumed $\left\langle Q_{\mathrm{ee}}\right\rangle$ to be equal to $\left\langle Q_{\mathrm{e} 1}\right\rangle$ (also following [50]).

\section{RESULTS AND DISCUSSION}

\section{A. Deviations from LTE}

In the literature there are numerous statements concerning LTE in argon plasmas, ranging from the existance of LTE to strong deviations from LTE (see, e.g., [18,21,52,53]). Controversial results can be found especially for the cathode region of electric arcs (see, e.g., [1,2,8,54-56]), even under comparable experimental circumstances. Thus we considered it appropriate to perform a close examination of evidence for departures from LTE in the cathode region of our low-current free-burning arc in argon under atmospheric pressure.

\section{Larenz maximum}

First we investigated the development of the Larenz maximum [48] in the near-cathode region. Figure 7 shows the radially resolved LEC for the Ar I line at $7147 \AA$ for the first five axial positions in front of the cathode.

Although the slightly varying radial position of the maximum could be explained even within the LTE model by assuming an appropriate distribution of the temperature, 


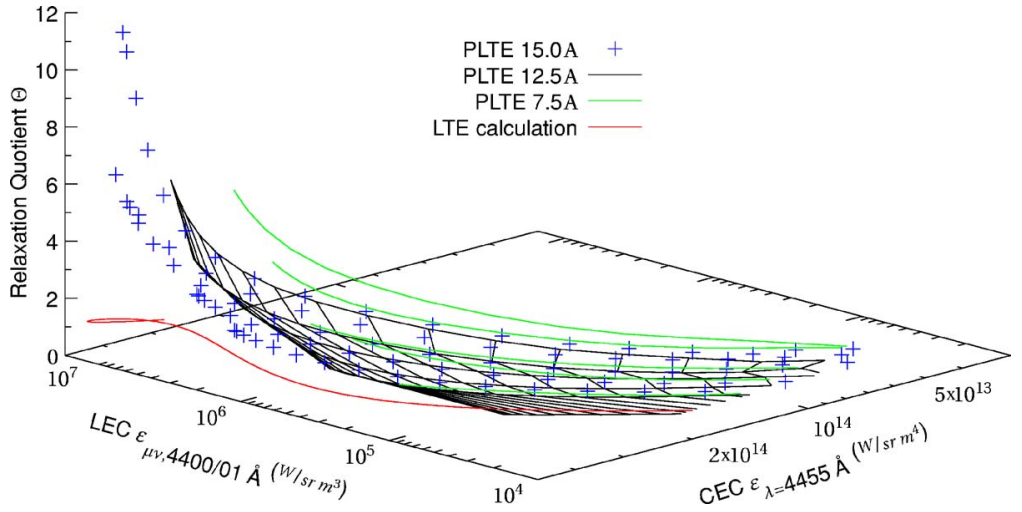

FIG. 10. (Color online) Extended, threedimensional Olsen-Richter plot showing measured Ar II LEC's, CEC's, and the relaxation quotient together with a calculated LTE curve. The measurements contain radial distributions (roughly from left to right, with the center of the discharge being in the upper left corner) for the first five axial positions in front of the cathode and three different currents (colors and line types). the more pronounced change in the absolute scale of the maximum is not covered by the LTE model. If the plasma is in LTE, the absolute value is constant and can be calculated from the atomic data of the transition to range around $170000 \mathrm{~W} / \mathrm{sr} \mathrm{m}^{3}$. The decreasing Larenz maximum, when approaching the cathode, is direct evidence for an increasing underpopulation of the excited Ar I levels. Similar observations have already been reported, e.g., in [1,6,7,52], for high-current arcs. Theoretical calculations describe this effect, too $[57,58]$.

\section{Relaxation method}

The increase of the line emission after an interruption of power as shown in Fig. 6 is also direct evidence of non-LTE phenomena, because it is not possible within the LTE model. The authors of [2,54-56] observed a similar increase in line emission during power interruption in the near-cathode region of high-current arcs. However, they did not attribute this to a non-LTE effect due to a difference between $T_{\mathrm{e}}$ and $T_{\mathrm{g}}$, but supposed a "natural" cooling according to LTE in combination with a line-of-sight effect because of a temperaturedependent radial shift of the Larenz maximum. They could not verify this experimentally as their setup did not yield spatially resolved data.

Figure 8 shows the Abel inverted LEC's measured with the relaxation setup for the first five axial positions in front of the cathode of our arc discharge. By comparing the profiles for emission in the continuous operation mode emission (solid symbols) with the profiles for the transient emission maximum during power interruption (open symbols) we note that the rise in line emission takes place along the entire profile. Thus we can rule out a cooling effect and a subsequent radial shift of the Larenz maximum as reason for the enhanced line emission. This is also consistent with our findings of deviations from LTE in front of a cathode operated in the diffuse mode [25]. We conclude that the rise in line emission during a power interruption is a manifestation of $T_{\mathrm{g}}$ being below $T_{\mathrm{e}}$ (and thus $\beta>1$ ).

\section{Olsen-Richter plots}

Another way to characterize deviations from LTE is the usage of so-called Olsen-Richter (OR) plots [59,60], which have been applied successfully to arc plasmas near the cathode tip before $[8,16,61]$. OR plots have some notable features.

(i) In principle, any two emission quantities can be used to construct an OR plot.

(ii) Due to their log-log scaling, OR plots are independent of all uncertainties in atomic data ( $A$ values, $\xi$ factors) and all other multiplicative error sources (detector calibration, etc.), because they all lead to a constant shift of the measured curve.

(iii) OR plots contain only directly measured data, so they are also independent of a concrete plasma model.

Figure 9 shows an OR plot with measured LEC's for Ar II lines and CEC's in dependence of the radial and axial position $(r, z)$ along with a calculated curve for LTE.

From Fig. 9 we can deduce that the deviations from LTE increase towards the cathode. It is not possible to map the

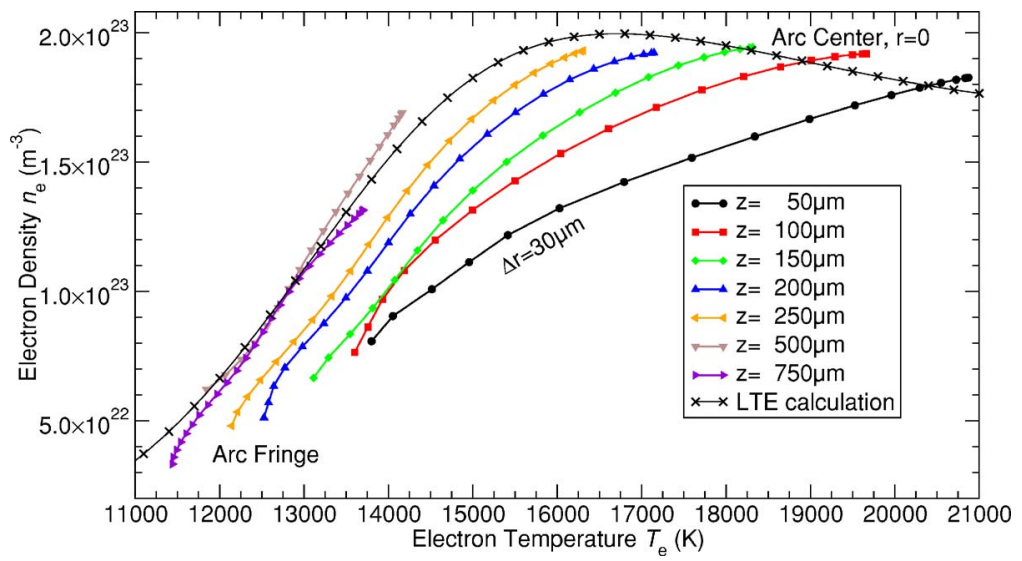

FIG. 11. (Color online) Electron densities and electron temperatures determined from the measurements presented in Fig. 9. 


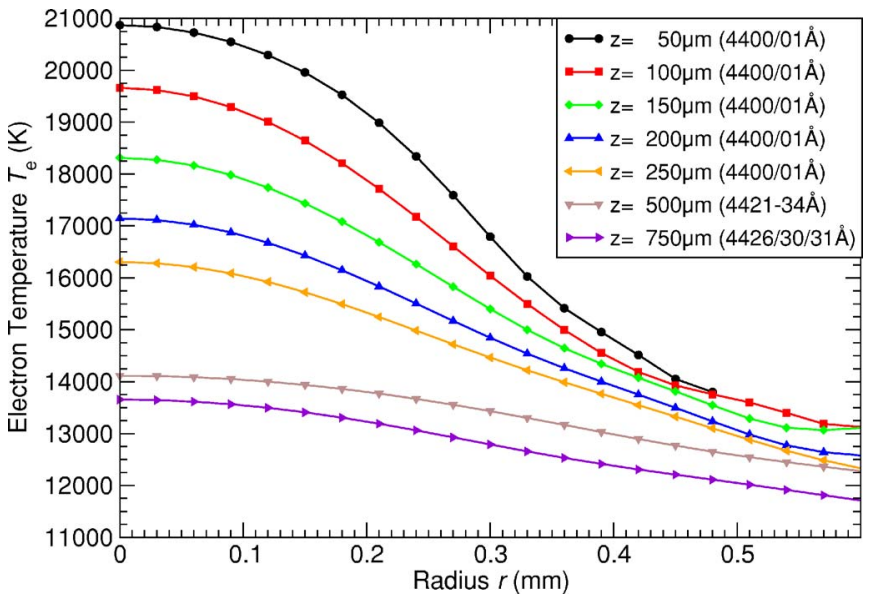

FIG. 12. (Color online) Radially resolved electron temperature profiles determined from the measurements presented in Fig. 9.

measured curves onto the LTE curve with a constant shift, so we can rule out experimental errors and uncertainties in atomic data as the reason for the deviations; their systematical increase towards the cathode could not be explained by these means anyway. Moreover, even the progression of the measured curves changes when approaching the cathode and it deviates from the LTE curve. We note that intersection points of the measured curves and the calculated LTE curve do not neccessarily denote an LTE plasma at this position. The LEC and CEC merely show the same magnitude as in the LTE case, but taking into account the radial variation, the plasma is clearly in PLTE. This is the same effect as described above for the PLTE calculations shown in Fig. 2, and it arises because the plasma state is not unambiguously determined by two measured emission quantities. However, the plasma appears to be in LTE for axial distances $\geqslant 500 \mu \mathrm{m}$. Finally, there is no physical curve that could be drawn through all measured data points for all distances. Therefore we conclude that one free parameter is not sufficient to explain these deviations; a plasma model describing the plasma needs at least two free parameters.

Since OR plots proved to be a very valuable tool to visualize and characterize the plasma, we extended the original OR concept by using an additional measured emission quan- tity: namely, the relaxation quotient $\Theta$. Apart from the axial and radial position $(r, z)$ we used the current $I$ as a third, experimentally alterable, implicit parameter. Figure 10 shows the three-dimensional, extended OR plot we constructed from our measurements. We did not use log scaling for the $\Theta$ axis in this case, because the values have a lower dynamic range than the emission coefficients and they are already free of multiplicative errors due to being a ratio of two measured emission quantities.

Apart from the obvious systematical deviations from LTE we can draw further conclusions from Fig. 10 in an analoguous extension of the arguments used above for Fig. 9: For no current can the measured points be connected to form a single physical curve, which means that in no case can the plasma be described using only one free parameter. Furthermore, the measured points for all currents cannot be connected to form a single hyperplane. Therefore even two free parameters are not sufficient to describe the plasma state in front of the cathode. This justifies the application of the PLTE plasma model that comprises three free parameters.

In the following we focus on one current $(I=12.5 \mathrm{~A})$. Further results for different currents can be found in [30].

\section{B. Electron density and electron temperature}

The electron densities and electron temperatures determined from the measured LEC's and CEC's from Fig. 9 are shown in Fig. 11, again together with a calculated LTE curve for comparison. The deviations from LTE in this diagram are completely analoguous to the OR plot in Fig. 9. Radially resolved temperature profiles of these measurements can be found in Fig. 12. Both Figs. 11 and 12 show steep radial and axial gradients for the electron temperature in front of the cathode, especially when leaving the region of the hot core. The maximum temperature is always located on axis and reaches $21000 \mathrm{~K}$ for a discharge operated with $12.5 \mathrm{~A}$ and a total voltage drop of about $13.5 \mathrm{~V}$. The distribution of the electron densitiy is displayed radially and axially resolved in Fig. 13 as a pseudocolor plot. Figures 11 and 13 show a relatively large on-axis region where the electron density is almost constant. Directly in front of the cathode a maximum value of about $2 \times 10^{23} \mathrm{~m}^{-3}$ is reached. This maximum value

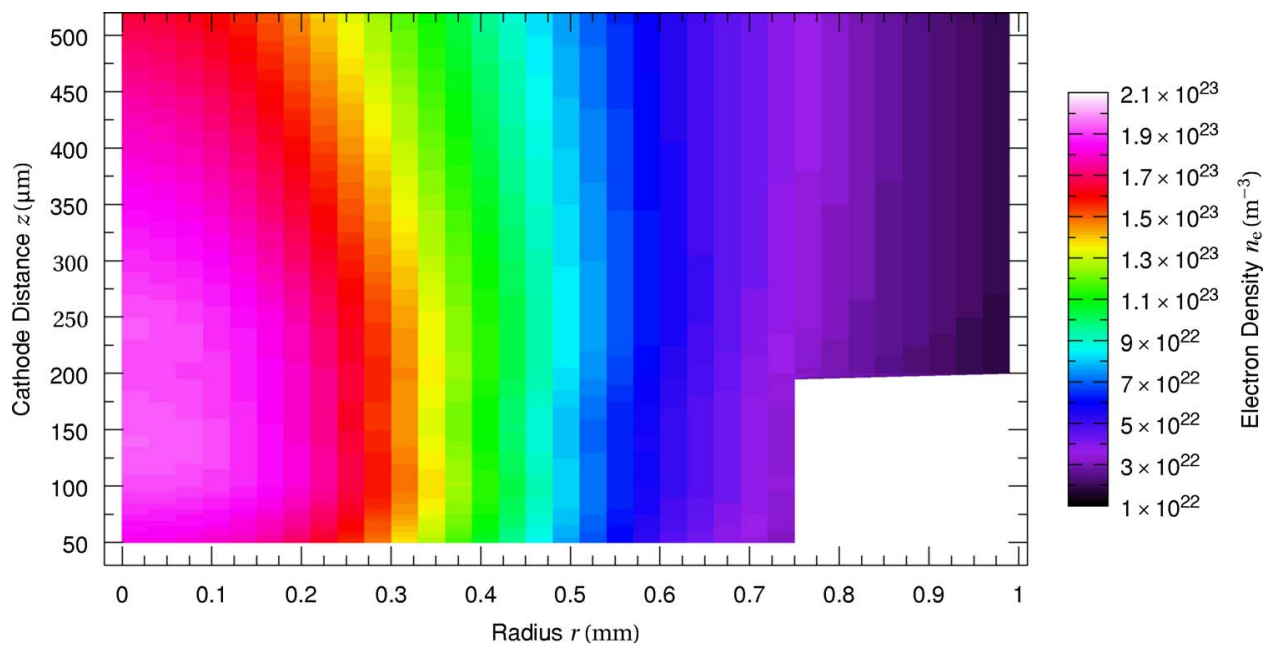

FIG. 13. (Color online) Pseudocolor plot of the radially and axially resolved electron density distribution determined from the measurements presented in Fig. 9. 


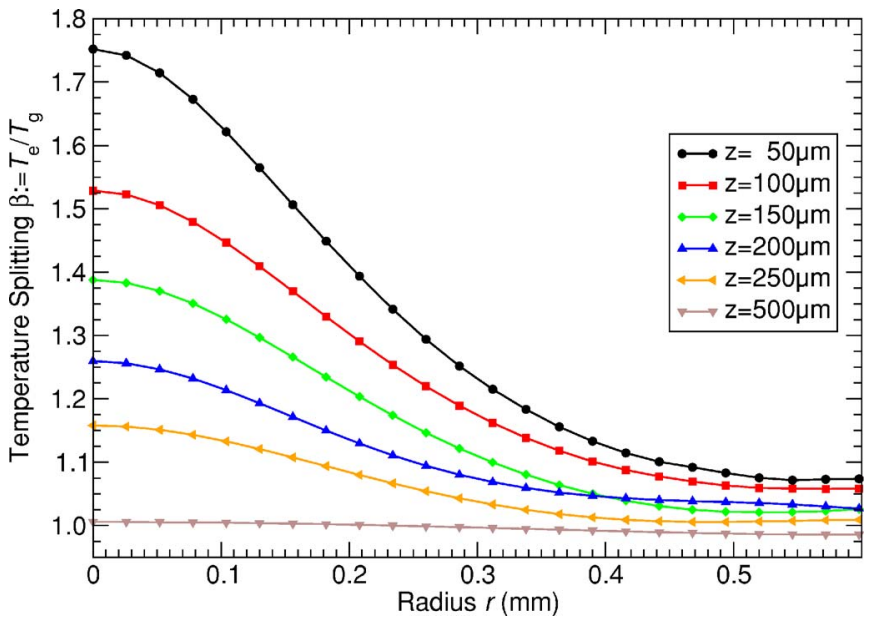

FIG. 14. (Color online) Radially resolved profiles for the temperature splitting $\beta$ determined from the relaxation experiment with Eq. (4) using values for $T_{\mathrm{e}}$ from Fig. 12.

is similar to the maximum value which can be calculated for the LTE case (see Fig. 11).

\section{Temperature splitting and gas temperature}

Together with the results from the relaxation experiment, which we performed in parallel to passive emission spectroscopy, we could determine radially and axially resolved values for the temperature splitting $\beta$ and thus the gas temperature $T_{\mathrm{g}}$. Figure 14 contains radially resolved profiles for $\beta$, which give a direct measure for the deviation from LTE. For $z \geqslant 500 \mu \mathrm{m}$ we could not detect any significant increase in line emission during the relaxation experiments, which leads to $\beta=1$ and indicates that the LTE state is reached. We note that $\beta$ (and thus the deviations from LTE) becomes larger when approaching the center of the discharge in radial direction as well as when approaching the cathode tip in axial direction. This is basically the same behavior we found earlier for the diffuse mode [25], although the values for $\beta$ are somewhat higher in the hot-core mode. This is a remarkable item, because at first sight one could think that higher electron densities should lead directly to higher collision rates and thus to smaller deviations from LTE and to smaller values of $\beta$. Obviously, this happens neither when comparing the two different discharge modes nor when looking at the radial and axial gradients. Even when increasing the current, which normally (but not neccessarily; see below) favors LTE in arc discharges, the deviations from LTE become larger, as can be seen in Fig. 10.

Figure 15 shows the corresponding radial and axial distribution of $T_{\mathrm{g}}$ as a pseudocolor plot. In contrast to the diffuse mode, where we found a slight radial and axial gradient leading to increasing values of $T_{\mathrm{g}}$ when approaching the cathode tip [25], the hot-core mode shows a distinct region directly in front of the cathode where $T_{\mathrm{g}}$ decreases.

Figures 14 and 15 augment our knowledge about the origin of the deviations from LTE. For high-current arcs the authors of $[7,8,38,58]$ attribute these deviations to a strong inward flux of cold atoms into the plasma column. The cur-

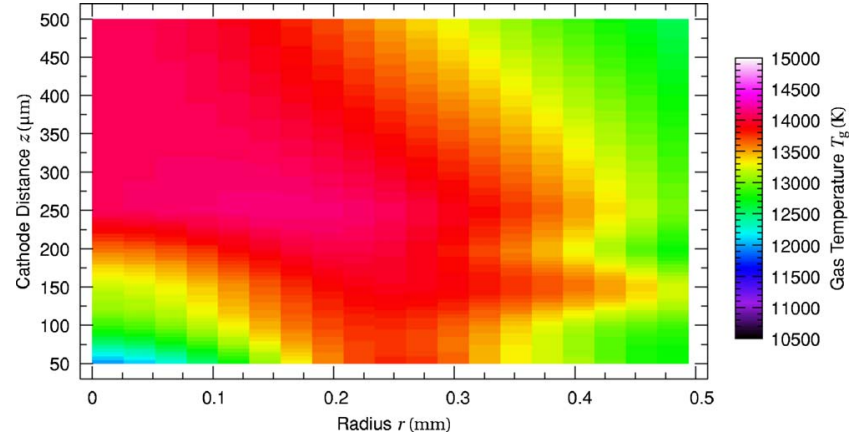

FIG. 15. (Color online) Pseudocolor plot of the radially and axially resolved gas temperature distribution determined from Fig. 14.

rent density $j$ at the cathode tip of our arc ranges approximately between 26 and $53 \mathrm{MA} / \mathrm{m}^{2}$ (corresponding to $I=7.5 \mathrm{~A}$ and $I=15 \mathrm{~A}$, and assuming that the current is solely emitted through the front surface of the cathode). Although this value is comparable to the current densities found in high-current arcs (e.g., in [58] current densities of 33 and $53 \mathrm{MA} / \mathrm{m}^{2}$ are given, depending on the affected cathode surface), the results found in these publications cannot be applied to our discharge directly. One reason for this is that the influx of the cold gas is driven by $\mathbf{a} \mathbf{j} \times \mathbf{B}$ force (with $\mathbf{B}$ being the magnetic field). As all relevant quantities (force, pressure, velocity, etc.) scale with $I j[62]$, we expect the pinch effect to play only a minor role in our discharge, which is operated around $10 \mathrm{~A}$, in comparison to a high-current arc, which is typically operated at $200 \mathrm{~A}$. Another difference that prevents a direct comparison is the shape of the cathodes, which is known to be crucial $[63,64]$. On the other hand, the pinch effect could explain the fact that the deviations from LTE become larger at higher currents (see Fig.10) and in comparison to the diffuse mode [25], where the current density $j$ is certainly smaller because of a larger emission area.

However, if the inward flux of cold atoms was the main driving force for the deviations in our discharge, we would expect to see at least some kind of thermalization taking

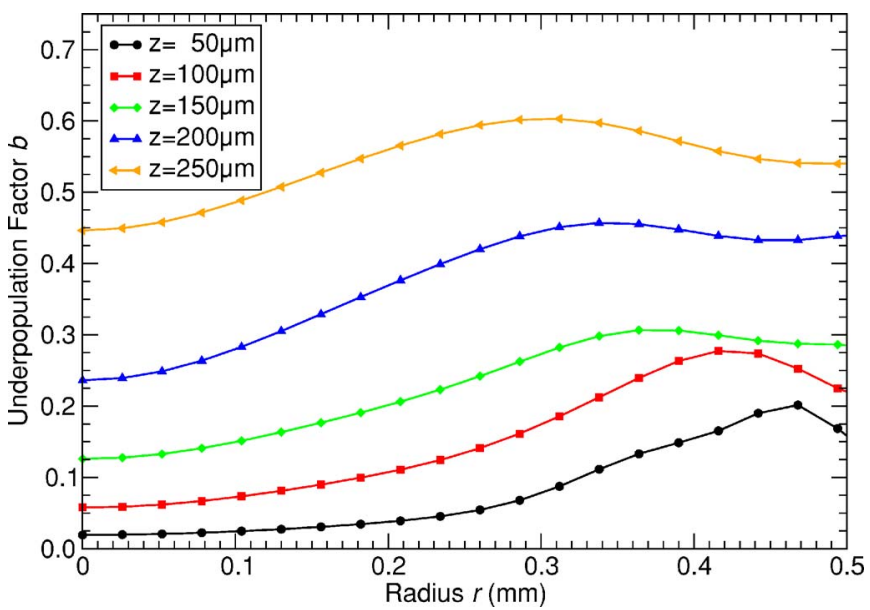

FIG. 16. (Color online) Radially resolved profiles for the underpopulation factor $b$ determined from the data presented in Fig. 11 and Fig. 14. 


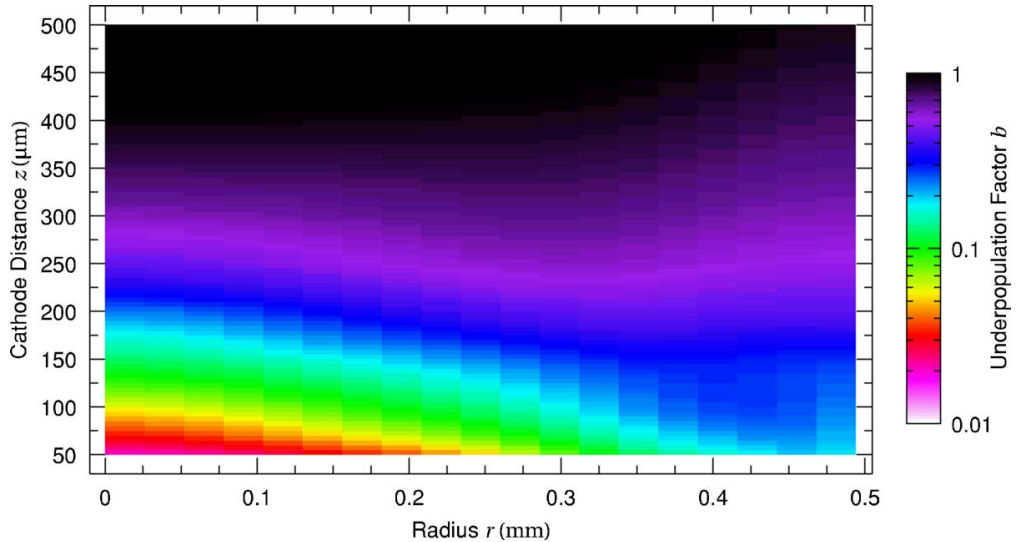

FIG. 17. (Color online) Pseudocolor plot of the radially and axially resolved distribution of the underpopulation factor according to Fig. 16. place along this way. This would include the fact that the gas temperature should be rising while cold atoms from the outside are streaming inwards. Thus, for the center of the cathode region there have to be additional effects driving the plasma away from LTE.

\section{Further derived plasma parameters}

Having determined a complete set of plasma parameters $\left(n_{\mathrm{e}}, T_{\mathrm{e}}, \beta\right)$, we used the PLTE model to calculate the underpopulation factor $b$. However, the results for $b$ presented in Fig. 16 should be taken as an indication of the order of magnitude only. As already mentioned in [24,29], the errors for $b$ are considerable because of the way the underpopulation is linked to the exponential factors in the SahaBoltzmann relations. Its uncertainity can quite easily exceed $50 \%$.

Figure 17 shows the results for $b$ as a radially and axially resolved pseudocolor plot. The strong axial gradient is especially remarkable. The underpopulation can reach values down to 0.01 directly in front of the cathode and is noticeably stronger compared to our results from the diffuse mode [25].

According to Eq. (5) we also calculated the radially and axially resolved distribution of the electric field strength $E$, displayed in Fig. 18 as a pseudocolor plot. These values likewise represent only an approximate estimate for $E$ as Eq. (5) contains a further uncertainity factor $\alpha \in[0.75-1.38]$ (see [65]) and does not account for the steep gradients. Nevertheless, Fig. 18 provides us with a rough overview of the magnitude of the electric field and its spatial distribution.

\section{SUMMARY, CONCLUSIONS, AND PROSPECTS}

We investigated the near-cathode region of a low-current free-burning arc in argon under atmospheric pressure. The arc was operated in the so-called hot-core mode, which is characterized by a hot plasma region emitting intense Ar II line radiation in front of the cathode. Using different methods (Larenz maximum, Olsen-Richter plots, relaxation method) we found that this region, approximately $500 \mu \mathrm{m}$ in dimension, shows noticeable deviations from LTE in spite of the high electron densities of about $2 \times 10^{23} \mathrm{~m}^{-3}$. Employing extended, three-dimensional Olsen-Richter plots we could also show that a PLTE plasma model with at least three free parameters is needed to describe the plasma. The application of spatially resolved passive and active emission spectroscopy allowed us to detetermine $n_{\mathrm{e}}, T_{\mathrm{e}}$, and $T_{\mathrm{g}}$ with radial and axial resolution and subsequently to calculate estimates for the underpopulation factor $b$ and the electric field strength

$E$. This gives a spatially resolved insight into the non-LTE plasma state in the hot core in front of free-burning arc cathodes.

The relaxation method in combination with passive emission spectroscopy as an independent spatially resolving plasma diagnostic technique for measuring $T_{\mathrm{e}}$ and $T_{\mathrm{g}}$ in this plasma regime is especially welcome, because the previously applied Thomson and Rayleigh scattering experiments

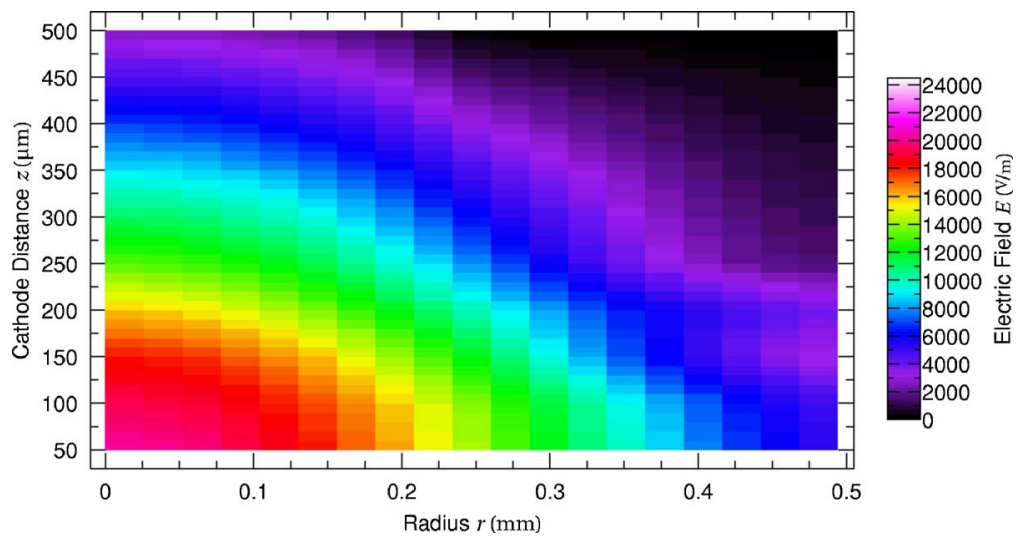

FIG. 18. (Color online) Pseudocolor plot of the radially and axially resolved distribution of the electric field strength according to Eq. (5). 
yielded questionable results and are still under discussion $[18,22,53,66,67]$. Furthermore, both laser scattering techniques are difficult to apply in the near-cathode region because of the large amount of stray light.

Obviously Griem's often-cited criterion for the establishment of LTE [68] is not applicable here. As it only covers the loss of energy by radiation, we can conclude in reverse that close to the cathode of our discharge other mechanisms of energy loss dominate. On the other hand, the inward drift of cold atoms, in the literature the supposed source for deviations from LTE in high-current arcs $[7,8,38]$, cannot explain our results satisfyingly either. We have started a coop- eration with our colleagues in Bochum $[13,69,70]$ to develop a simulation that shall reproduce our measured plasma parameters and will hopefully clarify which physical processes are dominant and drive the plasma away from LTE in the vicinity of the cathode.

\section{ACKNOWLEDGMENTS}

We thank Osram for providing us with the electrode material, K. Danzmann for permitting the completion of this work at the AEI, and M. Heurs for a critical reading of the manuscript.
[1] G. N. Haddad and A. J. D. Farmer, J. Phys. D 17 (1984).

[2] D. Wei and R. J. Kearney, J. Quant. Spectrosc. Radiat. Transf. 46, 537 (1991).

[3] S. Pellerin, K. Musiol, B. Pokrzywka, J. Chapelle, and A. Czernichowski, J. High Temp. Chem. Processes 1, 487 (1992).

[4] S. C. Snyder, G. D. Lassahn, and L. D. Reynolds, Phys. Rev. E 48, 4124 (1993).

[5] S. Pellerin, K. Musiol, B. Pokrzywka, and J. Chapelle, J. Phys. D 27, 522 (1994).

[6] J. Haidar, J. Phys. D 28, 2494 (1995).

[7] M. Razafinimanana, P. Gudzy, A. Gleizes, L. E. Hamidi, and S. Vaquié, J. High Temp. Chem. Processes 4, 51 (1995).

[8] B. Pokrzywka, K. Musioł, S. Pellerin, E. Pawelec, and J. Chapelle, J. Phys. D 29, 2644 (1996).

[9] B. Pokrzywka, S. Pellerin, K. Musiol, F. Richard, and J. Chapelle, J. Phys. D 29, 2841 (1996).

[10] J. Lowke, R. Morrow, and J. Haidar, J. Phys. D 30, 2033 (1997).

[11] S. Pellerin, K. Musiol, B. Pokrzywka, E. Pawelec, F. Richard, J. Cormier, and J. Chapelle, High Temp. Mater. Processes (N.Y., NY, U.S.), 1, 249 (1997).

[12] H. Schmitz and K.-U. Riemann, J. Phys. D 35, 1727 (2002).

[13] S. Lichtenberg, L. Dabringhausen, O. Langenscheidt, and J. Mentel, J. Phys. D 38, 3112 (2005).

[14] M. Redwitz, O. Langenscheidt, and J. Mentel, J. Phys. D 38, 3143 (2005).

[15] J. Reiche, F. Könemann, W. Mende, and M. Kock, J. Phys. D 34, 3177 (2001).

[16] F. Könemann, G. Kühn, J. Reiche, and M. Kock, J. Phys. D 37, 171 (2004).

[17] S. C. Snyder and R. E. Bentley, J. Phys. D 29, 3045 (1996).

[18] R. E. Bentley, J. Phys. D 30, 2880 (1997).

[19] G. Gregori, J. Schein, P. Schwendinger, U. Kortshagen, J. Heberlein, and E. Pfender, Phys. Rev. E 59, 2286 (1999).

[20] G. Gregori, U. Kortshagen, J. Heberlein, and E. Pfender, Phys. Rev. E 65, 046411 (2002).

[21] A. B. Murphy, Phys. Rev. Lett. 89, 025002 (2002).

[22] A. B. Murphy, Phys. Rev. E 69, 016408 (2004).

[23] J. Richter, in 10th International Conference on the Phenomena of Ionized Gases, edited by R. N. Franklin(Parsons, Oxford, 1971), Chap. 10 (http://www.tib.uni-hannover.de)

[24] J. Richter, , J. Phys. (Paris), Colloq. C3 (1980).

[25] G. Kühn and M. Kock, J. Phys. D 39, 2401 (2006).
[26] P. Bässler, Ph.D. thesis, Universität Hannover, Institut für Plasmaphysik, 1978.

[27] P. Bässler and M. Kock, J. Phys. B 13, 1351 (1980).

[28] K. P. Nick, Ph.D. thesis, Christian-Albrechts-Universität zu Kiel, Mathematisch-Naturwissenschaftliche Fakultät, 1982.

[29] K.-P. Nick, J. Richter, and V. Helbig, J. Quant. Spectrosc. Radiat. Transf. 32, 1 (1984).

[30] G. Kühn, Ph.D. thesis, Universität Hannover, Logos Verlag (Berlin), 2005.

[31] W. L. Wiese, J. W. Brault, K. Danzmann, V. Helbig, and M. Kock, Phys. Rev. A 39, 2461 (1989).

[32] V. Vujnović and W. L. Wiese, J. Phys. Chem. Ref. Data 21, 919 (1992).

[33] S. Pellerin, K. Musiol, K. Dzierzega, and J. Chapelle, J. Quant. Spectrosc. Radiat. Transf. 57, 359 (1997).

[34] E. Schulz-Gulde, Z. Phys. 230, 449 (1970).

[35] D. Hofsaess, J. Quant. Spectrosc. Radiat. Transf. 19, 339 (1978)

[36] S. E. Schnehage, M. Kock, and E. Schulz-Gulde, J. Phys. B 15, 1131 (1982).

[37] A. T. M. Wilbers, G. M. W. Kroesen, C. J. Timmermans, and D. C. Schram, J. Quant. Spectrosc. Radiat. Transf. 45, 1 (1991).

[38] B. Pokrzywka, K. Musiol, S. Pellerin, E. Pawelec, J.-M. Cormier, and J. Chapelle, C. R. Acad. Sci. Paris 327, 391 (1999).

[39] G. Kühn, F. Könemann, and M. Kock, J. Quant. Spectrosc. Radiat. Transf. (to be published).

[40] G. Pretzler, Z. Naturforsch., A: Phys. Sci. 46, 639 (1991).

[41] G. Pretzler, H. Jäger, T. Neger, H. Philipp, and J. Woisetschläger, Z. Naturforsch., A: Phys. Sci. 47, 955 (1992).

[42] M. Kock and J. Richter, Ann. Phys. 24, 30 (1969).

[43] D. B. Gurevich and I. V. Podmoshenskii, Opt. Spectrosc. 15, 319 (1963).

[44] H. Kafrouni, Physica B \& C 98, 100 (1979).

[45] J. A. M. van der Mullen, D. A. Benoy, F. H. A. G. Fey, B. van der Sijde, and J. Vlcek, Phys. Rev. E 50, 3925 (1994).

[46] F. H. A. G. Fey, W. W. Stoffels, J. A. M. van der Mullen, B. van der Sijde, and D. C. Schram, Spectrochim. Acta, Part B 46, 885 (1991).

[47] H. R. Griem, Principles of Plasma Spectroscopy (Cambridge University Press, Cambridge, England, 2005).

[48] R. W. Larenz, Z. Phys. 129, 327 (1951). 
[49] W. Finkelnburg and H. Maecker, in Handbuch der Physik: Gasentladungen II, edited by S. Flügge (Springer-Verlag, Berlin, 1956), Vol. XXII, pp. 408-409.

[50] M. Mitchner and C. H. Kruger, Partially Ionized Gases (Wiley, New York, 1973).

[51] R. S. Devoto, Phys. Fluids 10, 354 (1967).

[52] A. J. D. Farmer and G. N. Haddad, Appl. Phys. Lett. 45, 24 (1984).

[53] S. C. Snyder, L. D. Reynolds, G. D. Lassahn, J. R. Fincke, C. B. Shaw, and R. J. Kearney, Phys. Rev. E 47, 1996 (1993).

[54] S. Vacquie and A. Gleizes, J. Quant. Spectrosc. Radiat. Transf. 49, 453 (1993).

[55] D. Wei and R. J. Kearney, J. Quant. Spectrosc. Radiat. Transf. 49, 455 (1993).

[56] P. Gudzy, M. Razafinimanana, and A. Gleizes, in Proceeding of the Fourth International Thermal Plasma Processes Conference, Athens, Greece, edited by P. Fauchais (Begell House, New York, 1997) (http://www.tib.uni-hannover.de)
[57] J. Haidar, J. Phys. D 30, 2737 (1997).

[58] J. Haidar, J. Phys. D 32, 263 (1999).

[59] H. N. Olsen, J. Quant. Spectrosc. Radiat. Transf. 3, 305 (1963).

[60] J. Richter, Z. Astrophys. 61, 57 (1965).

[61] F. Könemann, Ph.D. thesis, Universität Hannover, 2000.

[62] H. Maecker, Z. Phys. 141, 198 (1955).

[63] P. Zhu and J. J. Lowke, J. Phys. D 26, 1073 (1993).

[64] J. Haidar and A. J. D. Farmer, J. Phys. D 27, 555 (1994).

[65] H. Maecker, Ergeb. Exakten Naturwiss. 25, 293 (1951).

[66] A. J. D. Farmer and G. N. Haddad, J. Phys. D 21, 426 (1988).

[67] A. B. Murphy and A. J. D. Farmer, J. Phys. D 25, 634 (1992).

[68] H. R. Griem, Phys. Rev. 131, 1170 (1963).

[69] L. Dabringhausen, O. Langenscheidt, S. Lichtenberg, M. Redwitz, and J. Mentel, J. Phys. D 38, 3128 (2005).

[70] F. H. Scharf and R. P. Brinkmann, J. Phys. D 39, 2738 (2006). 